\title{
Bacterial and Yeast Endophytes from Poplar and Willow Promote Growth in Crop Plants and Grasses
}

\author{
Zareen Khan, Grant Guelich, Ha Phan, Regina Redman, and Sharon Doty \\ School of Environmental and Forest Sciences, College of the Environment, University of Washington, Seattle, WA 98195-2100, USA \\ Correspondence should be addressed to Sharon Doty, sldoty@uw.edu
}

Received 27 April 2012; Accepted 22 July 2012

Academic Editors: A. D. Arencibia, M. Chodak, E. Perez-Artes, and S. Tsushima

Copyright () 2012 Zareen Khan et al. This is an open access article distributed under the Creative Commons Attribution License, which permits unrestricted use, distribution, and reproduction in any medium, provided the original work is properly cited.

Endophytic associations with plants have a beneficial effect for many different plant species with some of them being host specific. Several endophytes isolated from poplar and willow were screened for their effects on commercially important crops including corn, tomato, pepper, squash, sunflower, and grasses. Most of these endophytes produce growth hormones such as indoleacetic acid (IAA) and have the nitrogenase gene required for nitrogen fixation. The effects of these isolates on plant growth and yield were evaluated under greenhouse conditions. We found that inoculated plants not only had better viability and earlier flowering and fruiting, they also had increased plant growth and fruit yields when grown in nitrogen-limited soil. In a particular variety of perennial rye grass, the endophytes increased the total nitrogen content of the plants, indicative of nitrogen fixation, in addition to promoting plant growth. The use of specific endophytes may be preferable to the use of chemical fertilizers because of the monetary and environmental costs, contributing to more sustainable agricultural systems.

\section{Introduction}

Agriculture has become increasingly dependent on chemical sources of nitrogen. Production of nitrogen fertilizers is a chemically expensive process and uses nonrenewable resources including petroleum. Synthetic fertilizers also are hazardous to the environment. Excessive use of fertilizer produces nitrous oxide (a potent greenhouse gas) through soil microbial activity on excess fertilizer or leaches into water systems causing anoxia and algal blooms. The use of nitrogen fertilizer is of great importance in production of crops, as nitrogen is the major factor limiting growth under most conditions. Despite nitrogen's abundance in the atmosphere, it is not present in the form that can be utilized by plants to become an integral component of proteins, nucleic acids, and other biomolecules [1]. Since agriculture is expected to move toward environmentally sustainable methods [2], much attention has been recently paid to natural methods of biological nitrogen fixation. Biological nitrogen fixation has been well demonstrated in the legume-rhizobium symbiosis. It is a highly regulated system where these specific bacteria are able to biologically convert the atmospheric dinitrogen gas into ammonia by using the enzyme nitrogenase and high levels of ATP. More recently, it was found that certain microbes (termed endophytes) living inside sugarcane, a nonleguminous crop, provided fixed nitrogen to the plant. Since that discovery, endophytes have been isolated from plant tissues such as seeds, roots, stems, and leaves of a wide variety of plants [3-5], and culture independent analysis showed that a high number of unculturable species also colonize plants endophytically [6]. Several endophytic bacterial strains have been shown to have beneficial effects on their host plants by production of plant growth enhancing chemicals such as indole acetic acid [7] or cytokinins [8] and protection against biotic and abiotic stresses [9]. Beneficial effects on plant growth may also be achieved by improved nutrient acquisition including nitrogen fixation [10-12]. As such, these properties have attracted agronomic interest. Similar to the rhizobium-legume symbiosis, endophytes fix atmospheric nitrogen by means of the enzyme nitrogenase which is oxygen sensitive. In the case of endophytes, it is not certain how the nitrogenase is protected from oxygen. In the case of the sugarcane endophyte, the rapid respiration from metabolism of high levels of sucrose in the stems resulted in a microaerobic environment needed for the nitrogenase enzyme [13]. Others have shown that endophytes may 
TABLE 1: Crop varieties used for the study.

\begin{tabular}{ll}
\hline Crop & Variety \\
\hline Corn (Zea mays) & $\begin{array}{l}\text { Sugar buns, Quickie, Honey and } \\
\text { Cream, Supersweet Jubilee }\end{array}$ \\
Tomato (Solanum lycopersicum) & $\begin{array}{l}\text { Glacier, Chocolate Cherry, } \\
\text { Oregon Spring, Tiger-like, Heinz }\end{array}$ \\
Pepper (Piper nigrum) & Lady Bell, Yum yum Gold \\
Squash (Cucurbita pepo) & Bush Baby \\
Sunflower (Helianthus annuus) & $\begin{array}{l}\text { Pacino } \\
\text { Grasses }\end{array}$ \\
& $\begin{array}{l}\text { Kentucky blue grass, perennial } \\
\text { rye grass, bent grass, hair grass }\end{array}$ \\
\hline
\end{tabular}

use physical barriers including exopolysaccharides, internal vesicles, or biochemical methods to exclude the oxygen [14]. Reports on nonsymbiotic nitrogen fixation by Acetobacter spp. in sugarcane [15], Herbaspirillum spp. in rice [16], and Azospirillum spp. in cereals [17] have demonstrated the importance of endophytic bacteria in plant production. Endophytic colonizing bacteria and fungi have been isolated from several food crops including sugarcane [18], rice [19], wheat [20], coffee [21], alfalfa [22], sweet corn, sugar beet, squash and cotton [23], sweet potato [24] sweet chili [25], pepper [6], tomato [26], and strawberry [27], and also from bioenergy plants like poplar and willow [28, 29]. Most of these endophytes were associated with disease suppression, stress tolerance, growth promotion, or providing fixed nitrogen to the plants. Exploiting nitrogen fixing endophytes in nonlegumes can be an attractive technology in improving crop growth without the need of chemically produced fertilizers.

The endophytes used for the current study were isolated from poplar and willow trees that grow vigorously alongside rivers in rocky substrates with low nutrient levels. The strains grew well in nitrogen limited media and contained the nitrogenase gene [29] and some improved the growth of turfgrasses and maize $[30,31]$. The effectiveness of these endophytes on growth enhancement of a variety of commercially important crops and grasses was tested in this study.

\section{Material and Methods}

2.1. Seeds. Seeds were purchased from Territorial seed company, OR, USA. Table 1 lists the different varieties used for the study.

2.2. Endophytes. Table 2 lists the different bacterial and yeast endophytes isolated from poplar and willow trees that were selected for the study. These endophytes were chosen based on their abilities to produce plant growth hormones such as indole acetic acid (IAA) and nitrogen fixing abilities [24, 29] (Doty, SL and Khan, Z unpublished).

2.3. Seed Sterilization and Germination. For laboratory and greenhouse studies, depending upon the plant species, seeds were surface sterilized with different concentrations $(2-3 \%)$ of sodium hypochlorite for varying lengths of time (10 $20 \mathrm{~min}$ ) (Table 3). Next, the seeds were rinsed 4-5 times with sterile-distilled water and germinated on $0.5 \%$ agar medium, maintained at $26^{\circ} \mathrm{C}-36^{\circ} \mathrm{C}$, and exposed to a $12 \mathrm{hr}$ fluorescent light regime. Only those varieties that had $100 \%$ germination were used for further studies.

2.4. Fertilizer. Commercially available fertilizer (Plant Marvel Nutrisystem, $20(\mathrm{~N}): 20(\mathrm{P}): 20(\mathrm{~K}))$ supplemented with $10 \mathrm{mM} \mathrm{CaCl}_{2}$ was used for these studies.

\subsection{Determination of Optimum Strains for Each Crop Plant.} A large pilot study was conducted to screen the effects of different endophytes either individually or in combination on different plant cultivars. Endophytes and combinations of endophytes that showed the highest level of benefits in regards to plant growth promotion and health were used for additional studies.

2.6. Inoculation with Endophytes. The selected endophytes were grown in NF-CCM medium [32] or MG/L medium [33] or yeast extract-peptone-dextrose (YPD) medium and were prepared at a final concentration of $\mathrm{OD}_{600}=0.1$. The germinated seedlings $(N=36-72)$ were transferred to a sterile container and incubated with the different endophyte solutions for $4-5 \mathrm{hrs}$, except tomato varieties that were incubated for $24 \mathrm{hrs}$. The uninoculated controls received the same treatment and were exposed to 4-24 hours to the appropriate media in the absence of endophytes.

\subsection{Greenhouse Screenings of Endophyte Effects under Nitro-} gen Stress. After inoculation endophyte(s) exposed and control (no endophyte) seedlings were planted into trays containing either 36 or 72 seedlings in cell-packs filled with low nitrogen soil-Sunshine mix \#2 (Steuber Distributing Co., WA, USA), the trays were placed in a greenhouse under controlled conditions with $30^{\circ} \mathrm{C} / 20^{\circ} \mathrm{C}$ day/night temperature and $14 / 10 \mathrm{hr} /$ day/night light cycle with $40-60 \%$ humidity. The seedlings received only water as needed. At the end of one month, plants were harvested, roots and shoots separated, and wet weight biomass (g) recorded.

For grasses, a different treatment protocol was adopted. Several varieties of perennial rye grass, Kentucky blue grass, bentgrass, and hair grass were surface sterilized and germinated as above. Only the varieties that had 100\% germination following the surface sterilization procedure were selected. For screening studies, surface sterile seeds (approx. $135 \mathrm{~g}$ ) were either planted in low-nitrogen soil (Sunshine mix \#2) for nitrogen stress or nutrient rich soil (Sunshine mix \#1) for water stress experiments, in trays with 18 cells.

Water Stress. For the water stress treatments, the grasses that were to undergo water stress were allowed to grow for three weeks before being deprived of water. The control for the water stress consisted of endophyte inoculated and uninoculated control plants that received water every three days. They received $2.5 \mathrm{~L}$ of water which was roughly the amount the grasses were using every three days by the third 
TABLE 2: List of bacterial and yeast endophytes originally isolated from poplar and willow trees used for these studies.

\begin{tabular}{lcc}
\hline Isolate & Plant source & Closest 16s rDNA match \\
\hline WP5 & Poplar (Populus trichocarpa) & Rahnella sp. CDC 298779 \\
WP1 & Poplar (Populus trichocarpa) & Rhodotorulagraminis \\
PTD1 & Poplar (Populus trichocarpa) & Rhizobium tropici \\
PTD3 & Poplar (Populus trichocarpa) & Rhodotorula mucilaginosa \\
WW5 & Willow (Sitka sitchensis) & Sphingomonasyanoikuyae \\
WW11 & Willow (Sitka sitchensis) & Sphingomonasyanoikuyae \\
COMBO 1 & Poplar (Populus trichocarpa) and Willow (Sitka sitchenses) & All above \\
COMBO 2 & Poplar (Populus trichocarpa) & Only endophytes isolated from poplar \\
\hline
\end{tabular}

TABLE 3: Surface sterilization conditions.

\begin{tabular}{lcc}
\hline Crop & $\begin{array}{c}\text { Exposure time } \\
(\mathrm{min})\end{array}$ & $\begin{array}{c}\text { Sodium hypochlorite concentration } \\
(\%)\end{array}$ \\
\hline Corn & 20 & 3 \\
Tomato & 10 & 2 \\
Pepper & 15 & 2 \\
Squash & 10 & 2 \\
Sunflower & 15 & 2 \\
Grass & 20 & 2 \\
\hline
\end{tabular}

week. The grasses that underwent the low water (drought) stress were stressed at the beginning of the third week and received the same $2.5 \mathrm{~L}$ of water, but only every sixth day for six weeks at which point the experiment was finished and plants harvested for biomass and total nitrogen analysis.

Nitrogen Stress. In order to study the results of endophytes impacting the growth and development of grasses that have various levels of nitrogen, grasses were distributed in three groups (Table 4). The first group was a control which has no nitrogen fertilizer supplement in both inoculated and uninoculated grasses. The second group received a full dose of $10 \mathrm{~L}$ water with nitrogen, the third group received a full dose of $10 \mathrm{~L}$ water with $1 / 5$ the amount of nitrogen of the second group.

Preparation of Endophyte Consortium and Inoculation of Grass Seedlings. Three poplar bacteria strains (PTD1, WPB, and WP19), one poplar yeast strain (WP1), and one willow bacteria strain (WW6) were grown overnight in either MG/L medium or NF-CCM medium or YPD medium and inoculated as a consortia at an $\mathrm{OD}_{600}=0.1$. After the seeds germinated in soil, $5 \mathrm{~mL}$ of the prepared inoculum was added to the seedlings in soil. Uninoculated controls received the same treatment but without any endophytes. Height and root formations were monitored along with overall health.

Root and Tiller Nitrogen Content. After harvesting for biomass, a subset of plants were dried in an oven at $100^{\circ} \mathrm{C}$ and the roots and shoots was ground in a miller (Wiley Mill), weighed, and analyzed on a CHN analyzer (Perkin Elmer, Model-2400) at the analytical services center at the
School of Environmental and Forest Sciences, University of Washington.

Colonization. Colonization was verified on a subset of plants $(N=6)$. The plant tissue was surface sterilized with $0.525 \%$ sodium hypochlorite solution for 10 minutes, rinsed several times with sterile water, and plated on either MG/L or YPD. Resulting microbial growth was identified using colony morphology, color, and microscopic techniques.

2.8. Taking the Best Performing Endophyte-Plant Partnership to Maturity to Assess Final Yields under Nitrogen Stress. A subset of plants that responded positively to the endophyte treatments was transplanted to 5 gallon pots containing lownitrogen soil (Sunshine \#2). The nitrogen treatments are listed in Table 4 . The pots were randomly placed in a greenhouse under controlled conditions at $30^{\circ} \mathrm{C} / 20^{\circ} \mathrm{C}$ day/night temperature and $14 / 10 \mathrm{hr}$ day/night light cycle with $40-$ $60 \%$ humidity. Heights were measured throughout the study period and flowering and fruiting were recorded. At harvest, the soil was carefully teased out of the root systems and the roots were thoroughly washed and rinsed several times with water and patted dry. Roots and shoots were separated and wet biomass weights determined. The fruits produced were counted and weighed to quantify the total fruit yield.

2.9. Statistical Analysis. P values were determined by Duncan's multiple-range test and data were analyzed using the SAS statistical software version 9.3 (SAS Institute Inc., Cary, NC, USA).

\section{Results}

3.1. Stage 1: Screening Results. Only the varieties that had $100 \%$ germination on agar plates were used for studies.

Corn. All the selected corn germinated well on agar plates except for the Supersweet Jubilee variety. After one month of screening with the different endophytes, all the varieties responded positively with increased biomass obtained in the endophyte-colonized plants compared to uninoculated control plants. However, only a statistically significant difference was achieved in the root $(N=6, P=0.0084)$ and shoot 
TABLE 4: Nitrogen regime.

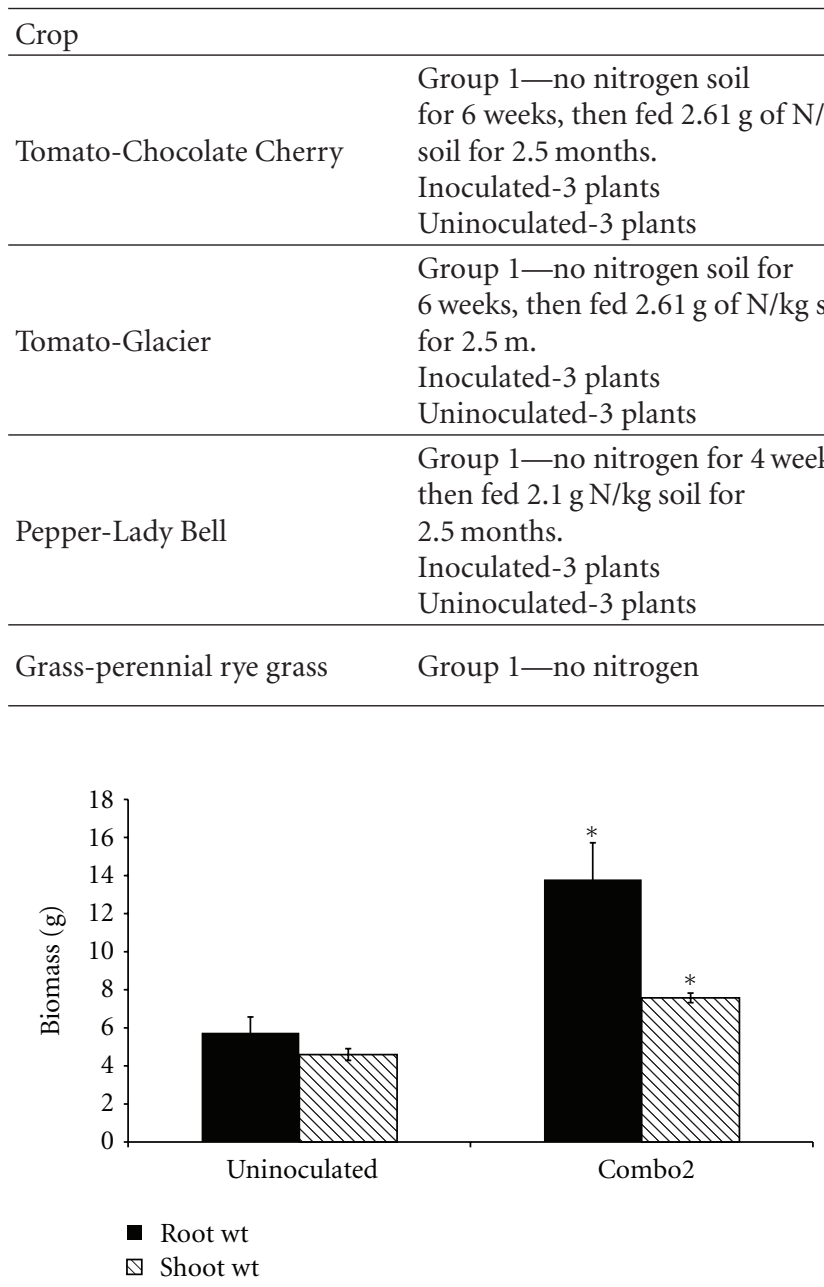

FIgURE 1: Total biomass of Quickie variety of corn. Uninoculated control and combo 2 inoculated plants showed significant differences in the root weights $(N=6, P=.0084)$ and in shoot weights $(N=6, P=0.003)$ at harvest. Significant difference $P<0.05$ is indicated by asterisk $(*)$.

weights $(N=6, P=0.003)$ of the Quickie corn variety with the Combo 1 endophyte treatment (Figure 1).

Tomato. For the tomatoes, different varieties responded differently to each endophyte treatment, with some suppressing seedling growth. However, in the Chocolate Cherry variety, some endophytes improved viability (WW5 (100\%), combo $1(68 \%)$, and WP5 (60\%)) whereas some had decreased viability (WP1 $(<50 \%)$, PTD1 $(<50 \%))$. Out of the ones that responded positively to the endophyte treatment, the WW5 (willow endophyte) inoculated plants looked healthier and greener than the uninoculated controls (Figure 2). After one month of growth in nitrogen poor soil, the WW5 inoculated plants had $68 \%$ more biomass when compared to the uninoculated control plants $(N=6, P<0.05)$ (Figure 3). For the Glacier variety, WP5 inoculated plants had the highest viability $(>90 \%)$ and produce more biomass
Nitrogen inputs

Group 2-low nitrogen-4 months in

no nitrogen soil receiving $11 \mathrm{~g}$ of $\mathrm{N} / \mathrm{kg}$ soil.

Inoculated-3 plants

Uninoculated-3 plants

Group 2-low nitrogen-4 months in no nitrogen soil

receiving $11 \mathrm{~g}$ of $\mathrm{N} / \mathrm{kg}$ soil.

Inoculated-3 plants

Uninoculated-3 plants

Group 2-low nitrogen-4 months in no nitrogen soil

receiving $6 \mathrm{~g} \mathrm{~N} / \mathrm{kg}$ soil

Inoculated-3 plants

Uninoculated-3 plants

Group 2-1/5 nitrogen-0.24 g N/kg Group 3-full soil nitrogen-1.2 g N/kg soil

the uninoculated controls (Figure 3). For the Tigerlike variety, analysis of percent biomass change showed a decrease in the root mass (50\%) in the uninoculated plants when exposed to the nitrogen stress $(N=6, P=0.0072)$ (Figure 3), whereas the PTD3 inoculated plants had higher root biomass. Organic Spring and Heinz, also responded positively to some of the endophytes (WW5, WW11, and PTD1), with a root promoting effect, although statistical analysis indicated it was not a significant difference.

Squash. Within two weeks of screening in poor nitrogen soil, the uninoculated plants started to exhibit signs of stress (wilting and chlorosis) while the inoculated controls stayed healthy for an additional month before displaying nitrogen stress (Figure 4).

Pepper. The Lady Bell variety with $100 \%$ germination on agar plates was screened with the different endophytes. The poplar yeast WP1 had a dramatic effect on the health, viability, and the biomass yield of endophyte-inoculated plants. As seen in Figure 5, WP1 inoculated plants had expanded 


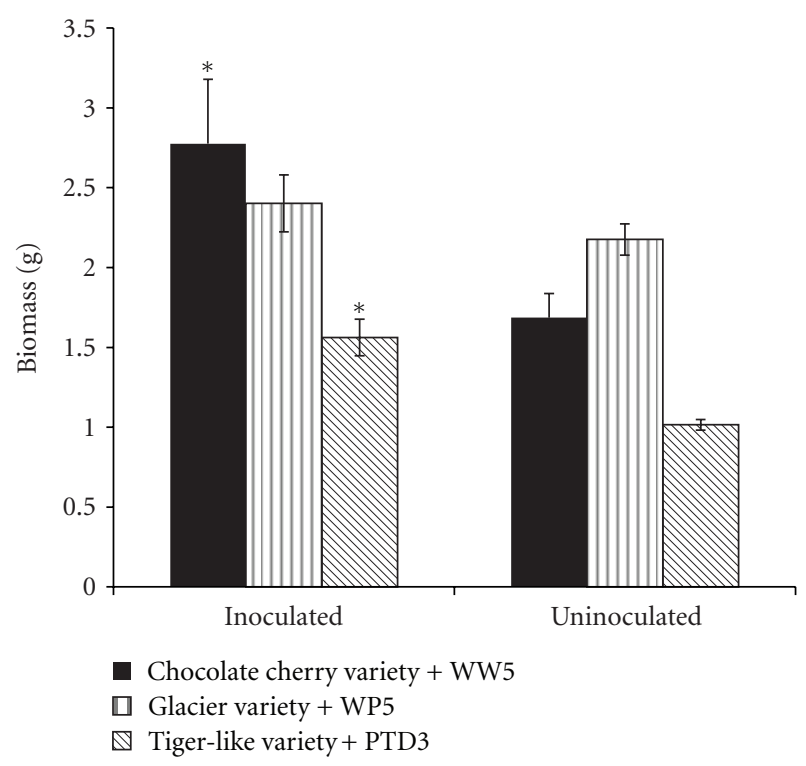

Figure 3: Total root weight of the three varieties of tomato. Total root weight of Chocolate Cherry variety, Glacier variety, and Tiger like variety of tomato. The data are shown as the mean \pm SEM from six plant samples. Significant differences were observed for Chocolate Cherry $(N=6, P<0.05)$ and Tiger Like varieties $(N=6, P=0.0072)$ at harvest. Significant difference $(P<0.05)$ is indicated by asterisk $\left(^{*}\right)$.

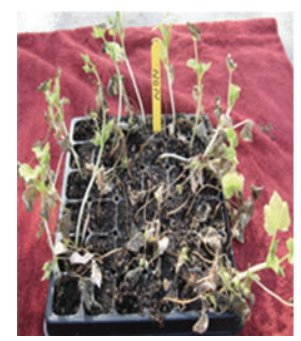

Uninoculated

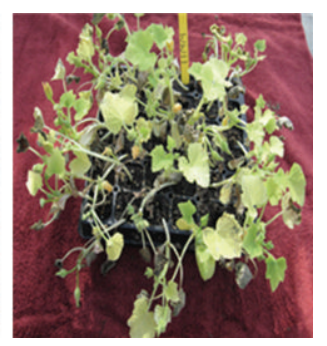

Inoculated with WP1

FIGURE 4: Growth response of inoculated and uninoculated squash plants after two weeks in nitrogen stress. The uninoculated plants suffered heavily due to lack of nitrogen, whereas the inoculated plants survived longer, indicating that the endophytes seemed to provide some protection to the plants from the nitrogen stress.

leaves and looked healthier and greener. A comparison of the root and shoot biomass in the inoculated and uninoculated plants revealed significant increases in root $(158 \%)$ and shoot $(137 \%)$ in the WP1 inoculated plants than in the uninoculated controls $(N=6, P \leq 0.0001)$ (Figures 6 and 7).

Sunflower. We did not notice any positive contribution by endophytes in plant growth development of the Pacino variety of sunflower (data not shown).

3.2. Stage 2: Final Yield. A subset of plant varieties that responded well in the screening study was transferred into 5 gallon pots containing low nitrogen soil and supplemented

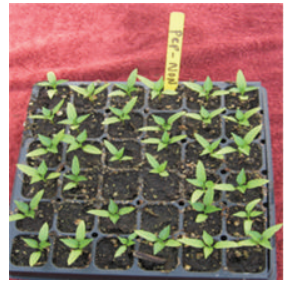

Uninoculated

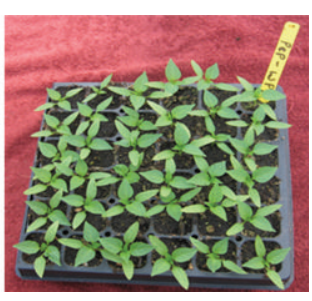

Inoculated with WP1
FIGURE 5: One-month-old uninoculated and inoculated pepper plants exposed to nitrogen stress. Plants inoculated with poplar yeast endophyte, WP1, look healthier, have expanded leaves, whereas the uninoculated control plants looked stunted and chlorotic.

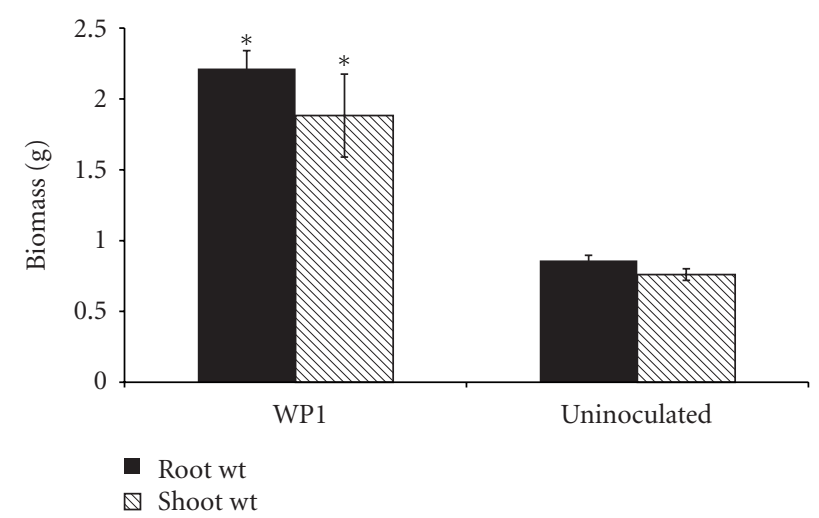

FIGURE 6: Root and shoot biomass of Lady Bell variety of pepper. Uninoculated control and WP1 inoculated plants showed significant differences in the root and shoot weights at harvest $(N=6$, $P<0.0001)$. Significant difference $(P<0.05)$ is indicated by asterisk $(*)$.

with different levels of nitrogen (Table 4) for a longer-term study.

\section{Tomato}

Chocolate Cherry Variety. In the first set that was grown for two months in nitrogen poor soils, WW5 inoculated plants were consistently taller (30\%) than the uninoculated controls. In the second set, after two months, a fertilizer was added and the experiment continued for another month before the plants were harvested. In both sets, the inoculated plants had a higher shoot weight compared to the uninoculated controls $(61 \%$ and $50 \%$, resp., in the two sets) (Figure 8). After 1.5 months, the number of flowers was counted, and the WW5 colonized plants had 120 flowers compared to the uninoculated counterparts that had only 76 flowers. The number of fruits produced at harvest was also higher for the inoculated plants (106 fruits) compared to the uninoculated plants (63 fruits). Using standard microbiological techniques, the presence of endophyte in WW5 colonized plants occurred in the vegetative tissue but not in the fruit and seed. 


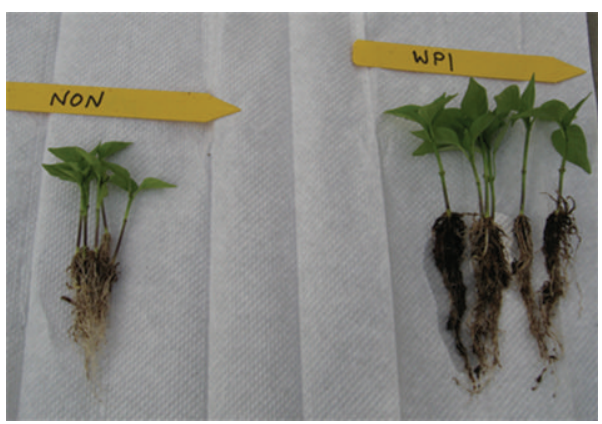

Uninoculated

Inoculated with WP1

FIGURE 7: Root and shoot biomass of pepper plants with or without WP1 after one month in soil lacking nitrogen. WP1 inoculated plants had a larger biomass compared to the uninoculated plants.

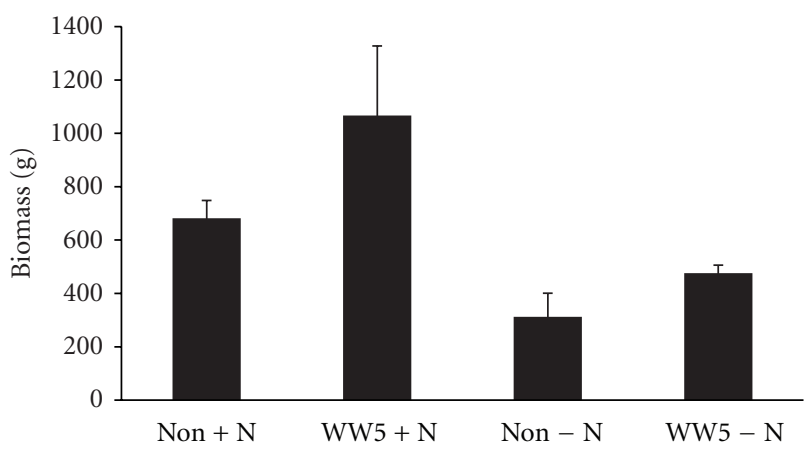

FIGURE 8: Shoot weight of tomato-chocolate Cherry variety. 2.5month-old shoot and root biomass of tomato varieties (Chocolate Cherry and Glacier) grown in low $(-)$ and high $(+)$ nitrogen soils. $(N=6, P<0.05)$.

Glacier Variety. Throughout the experiment, WP5 inoculated plants looked healthier and taller than the uninoculated control plants after transplanting in low-nitrogen and highnitrogen soil conditions. Biomass was measured at the end of 2.5 months and there were significant differences in both the root and shoot weights of the inoculated plants compared to uninoculated plants, with WP5 plants being larger for root and shoot biomass. Interestingly, for the plants that received nitrogen from the beginning, the difference was not very significant in the shoot weight, but more pronounced in the root weight (>16\%) (Figure 9). Similar to the Chocolate Cherry variety, the WP5 colonized plants also flowered earlier (108 flowers in 1.5 months) producing more fruits (95 fruits) at harvest compared to the uninoculated plants (65 flowers, 43 fruits). No endophyte was reisolated from the fruit or seed.

\section{Pepper}

Lady Bell Variety. After 1 month of screening in low-nitrogen soil, plants were then transplanted to 5 gallon pots containing either low-nitrogen or high-nitrogen soil. In this crop plant the effect of the endophyte was significant only in plants that were deprived of nitrogen, with the inoculated plants

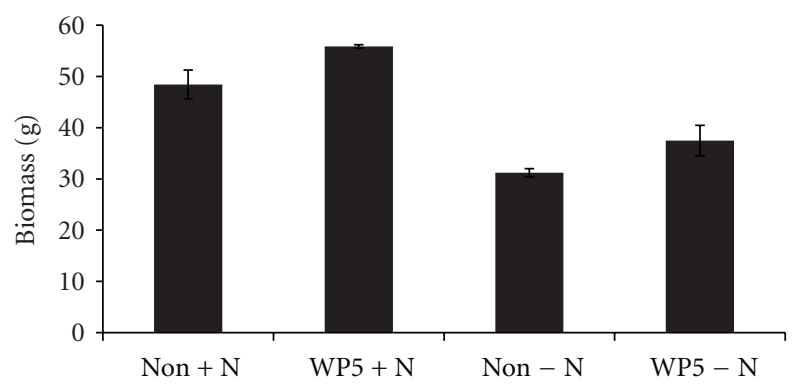

FIGURE 9: Root weight of tomato-Glacier variety. 2.5-month-old shoot and root biomass of tomato varieties (Chocolate Cherry and Glacier) grown in low $(-)$ and high $(+)$ nitrogen soils. $(N=6$, $P<0.05)$.

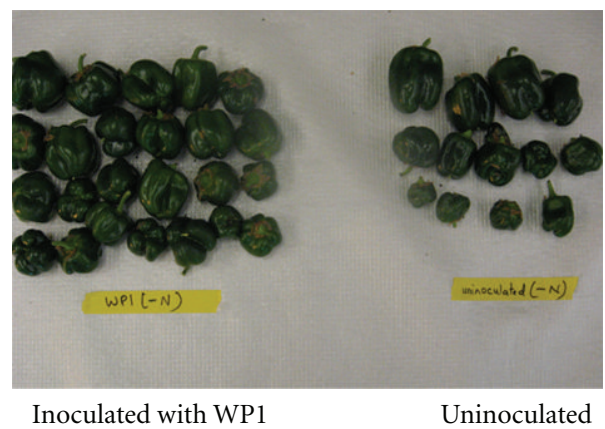

FIGURE 10: Fruit yield of Lady Bell variety of pepper at harvest after 4 weeks of growth in low nitrogen soil. WP1 inoculated peppers produced more fruit than the uninoculated control plants.

looking more robust and green, taller, flowering earlier (6 flowers in 1.5 months), and producing more fruits (24) than the uninoculated control plants ( 3 flowers in 1.5 months; 13 fruits final) (Figure 10). The biomass was measured at the end of 2.5 months. Endophyte-inoculated plants showed a higher \% biomass change in root (58.8\%) and shoot ( $>60 \%$ ) tissues, respectively, in nitrogen-stressed plants compared to the uninoculated plants (Figure 11). Using methods described above, the presence of the endophyte was confirmed in the vegetative tissue (lower stem and leaves) but was not found in either the fruit or seed. Enhancement of plant growth and biomass yield by WP1 was strongly observed in this particular variety of pepper.

\section{Grasses}

(a) Water Stress. Only the Brightstar SLT (G3) (Perennial rye grass) was chosen for further studies as the others did not respond well to the endophyte treatments. The grasses grew consistently until the drought stress was employed for a few days. The lack of water inhibited the growth rate slightly and the grass became dry, especially the uninoculated controls. At the last measurement, the endophyte-inoculated plants that were water stressed were taller than the controluninoculated 


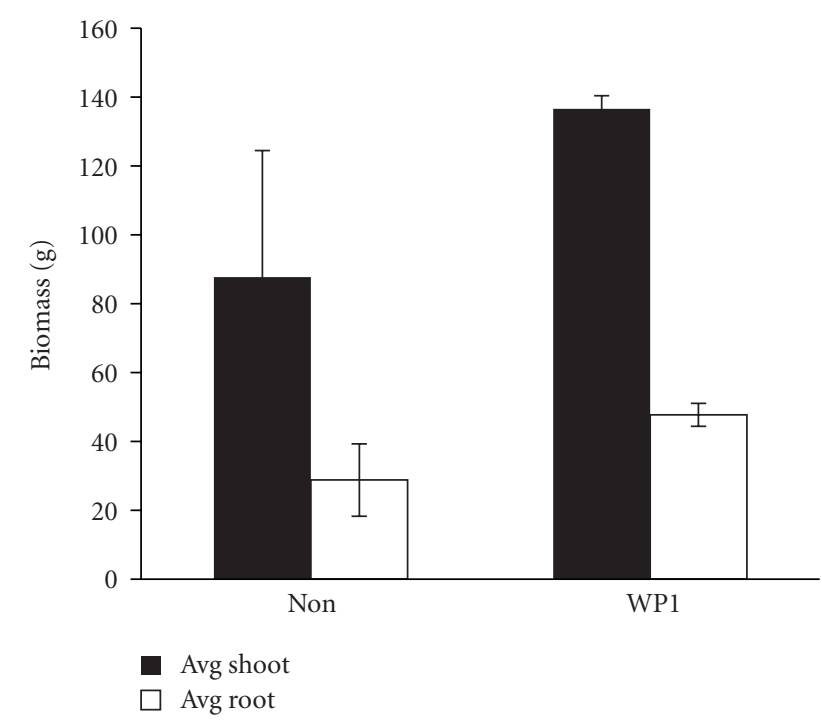

Figure 11: Shoot and root weight of Pepper-Lady Bell variety. Biomass differences of Lady Bell variety of pepper after four weeks in low nitrogen soil. WP1 inoculated plants had higher shoot and root weights compared to the uninoculated counterparts. Bars represent standard error of means.

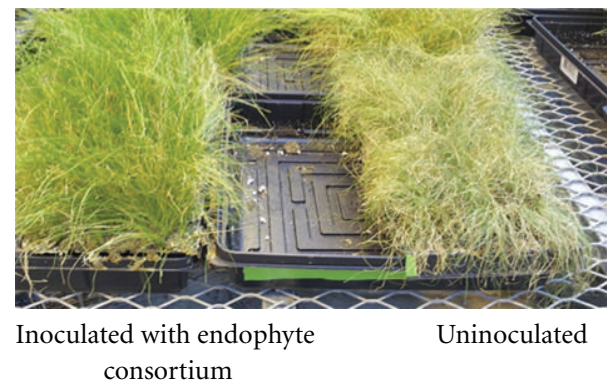

FIGURE 12: Five-week-old perennial rye grass (G3) responding to water stress. Uninoculated grasses look water stressed and chlorotic, whereas the inoculated (endophytes:PTD1, WPB, WP19, WP1, and WW6) grasses stayed greener longer.

plants that received ample water. While being exposed to low water stress (drought stress), the different treatments demonstrated a visible difference while undergoing the stress. As drought stress was imposed, uninoculated control grasses dried earlier and looked chlorotic when compared to endophyte-inoculated grasses, which stayed healthier and greener (Figure 12). At harvest, the biomass measurements revealed clear differences in root masses of inoculated and uninoculated grasses (Figure 13) with endophyte-colonized plants being larger than uninoculated control grasses. The endophyte-inoculated grasses formed thicker and longer roots than the uninoculated controls. Figures 14 and 15 show significantly higher root (60\%) and shoot biomass (48\%) produced in the inoculated grasses than the uninoculated controls. Overall, the endophyte-inoculated rye grass had more root mass, greater average weights, longer roots, taller shoots and survived longer when water stressed.

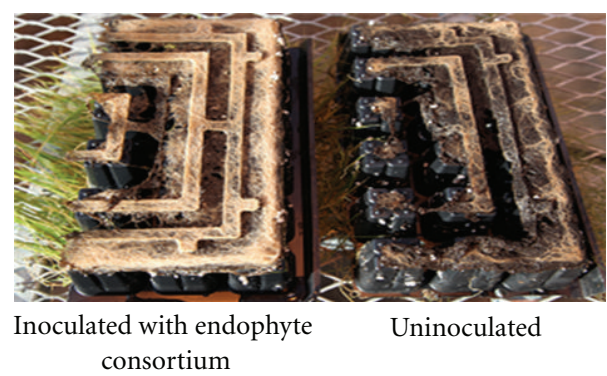

FIGURE 13: Underside of the grass trays. Inoculated (endophytes: PTD1, WPB, WP19, WP1, and WW6) grasses displaying more root mass than their uninoculated counterparts.

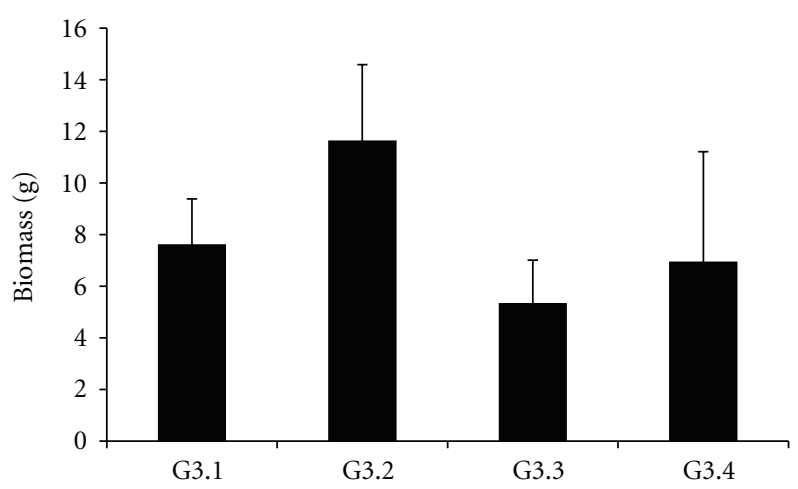

FIGURE 14: Root weights of inoculated and uninoculated grass exposed to water stress. Root weights of perennial grass variety (G3) inoculated with endophytes:PTD1, WPB, WP19, WP1, and WW6 and uninoculated exposed to low water stress (drought) at 5 weeks. G3.1-uninoculated (No stress), G3.2-inoculated (no stress), G3.3uninoculated (water stress), and G3.4-inoculated (water stress).

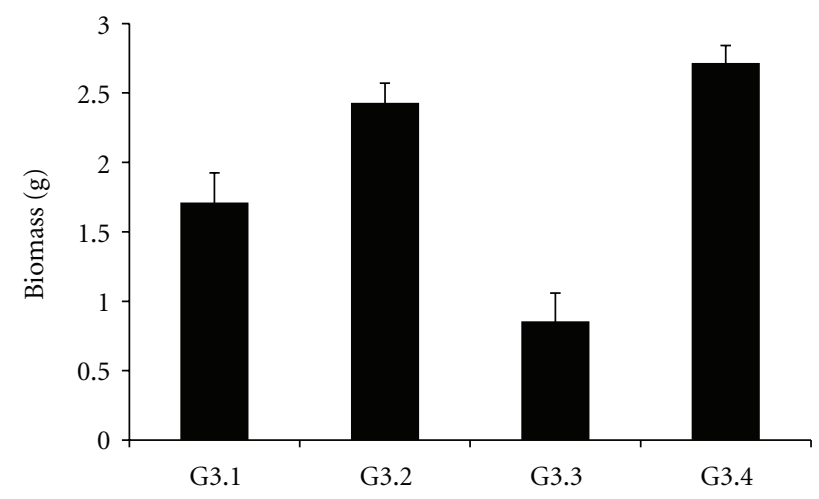

FIGURE 15: Shoot weights of inoculated and uninoculated grass exposed to water stress. Tiller biomass of perennial grass variety (G3) inoculated with endophytes:PTD1, WPB, WP19, WP1, WW6 and uninoculated exposed to low water stress (drought) at 5 weeks.. G3.1-uninoculated (no stress), G3.2-inoculated (no stress), G3.3uninoculated (water stress), and G3.4-inoculated (water stress). Bars represent standard error of means. 


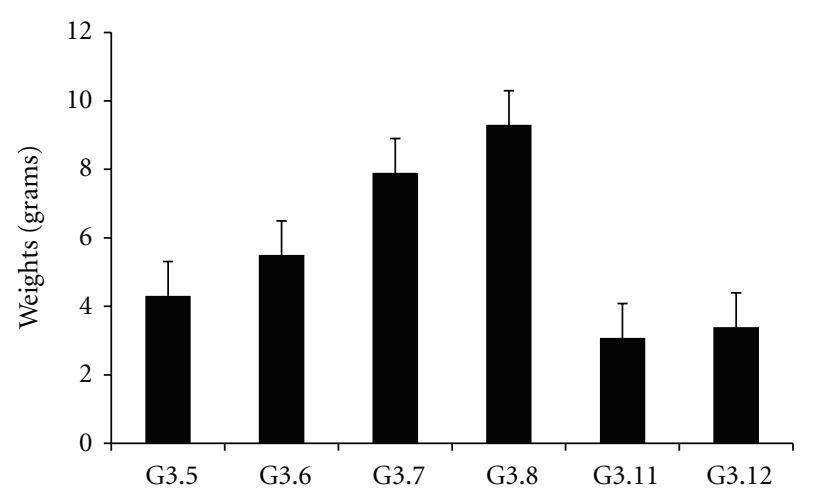

FIGURE 16: Root weights of inoculated and uninoculated grass under nitrogen stress. Root weights of perennial grass variety (G3) inoculated with endophytes:PTD1, WPB, WP19, WP1, and WW6 and uninoculated under nitrogen stress at 7 weeks. G3.5uninoculated (no nitrogen), G3.6-inoculated (no nitrogen), G3.7uninoculated (full nitrogen), G3.8-inoculated (full nitrogen), G3.11-uninoculated (1/5 nitrogen), G3.12-inoculated and (1/5 nitrogen). Bars represent standard error of means.

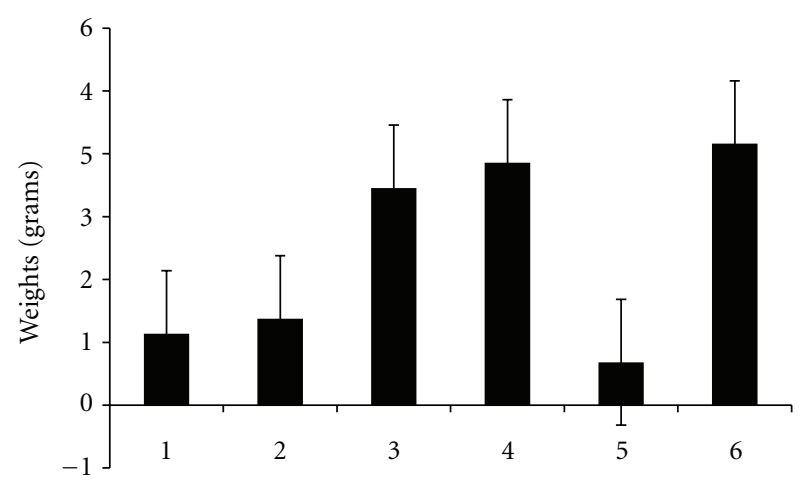

Figure 17: Tiller biomass of Perennial rye grass variety (G3) inoculated with endophytes-PTD1, WPB, WP19, WP1, WW6) and uninoculated controls under nitrogen stress at 7 weeks. 1-Uninoculated (No nitrogen), 2-Inoculated (No nitrogen), 3Uninoculated (Full nitrogen), 4-Inoculated (Full nitrogen), 5Uninoculated (1/5 nitrogen), 6-Inoculated (1/5 nitrogen). Bars represent standard error of means.

(b) Nitrogen Stress. The average heights were somewhat consistent across all treatments until the fertilizers were added. The variations in heights were marginal when the plants received no nitrogen or a full dose, but the group that received $1 / 5$ of the nitrogen supplementation displayed significant size differences in the inoculated compared to control plants. At the end of the experiment, the inoculated grasses that received the limited quantity of nitrogen $(1 / 5)$ were significantly taller than uninoculated control plants. Interestingly, the greatest root and shoot weights (Figures 16 and 17) occurred in endophyte-inoculated grasses that received 1/5 concentration of nitrogen fertilizer. A 425\% increase in the shoot weights was recorded for the inoculated grasses that were given $1 / 5$ amount of nitrogen supplement compared to the uninoculated counterparts.

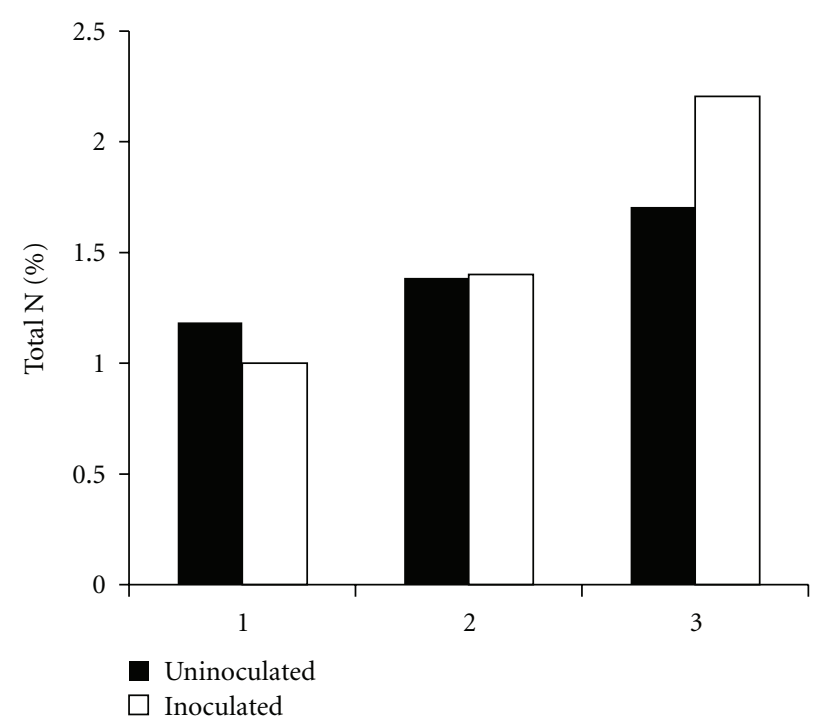

FIGURE 18: Perennial rye grass total nitrogen in tillers. Total nitrogen content found in the tillers of inoculated with endophytes-PTD1, WPB, WP19, WP1, and WW6 and uninoculated perennial grass variety $(\mathrm{G} 3)$ at 7 weeks in the various nitrogen treatments. 1: no nitrogen, 2: full nitrogen, and 3: 1/5 nitrogen.

(C) Total Nitrogen Content. As shown in Figures 18 and 19 , inoculated plants had, in general, more nitrogen incorporated in the treatment group 2. Significant higher levels of $\mathrm{N}(26 \%)$ were observed in endophyte-colonized shoot samples exposed to $1 / 5 \mathrm{~N}$ regime. All other treatments did not show significant differences in shoot $\mathrm{N}$ levels compared to uninoculated controls. In the roots, all of the endophytecolonized plants for all $\mathrm{N}$ regimes (low to high) were larger than uninoculated controls, with a dramatic increase of $203 \%$ in the total nitrogen.

\section{Discussion}

Endophytes affect plants differently, but overall they do make differences in the root system development, improving the general health and providing stress tolerance. In our studies we found that the endophytes had a broad host range, colonizing monocots as well as dicots, increased seed germination, and enhanced plant survival and stress tolerance to low nitrogen and low water stress. The endophyte treatment also promoted a growth response resulting in increased fruit yields. In grasses, we found evidence of nitrogen fixation as seen by an increase in the nitrogen content of endophytecolonized roots. In some plant lines there was more growth in the roots and by far, the most common mechanism that endophytes use to stimulate root growth is through secretion of auxin within the plant. Indole-3-acetic acid (IAA) is the most common plant auxin that induces initiation of lateral and adventitious roots and root hairs which increase the root surface area. Since some of the chosen endophytes have been shown exogenously to produce IAA, better root growth observed may be attributed to the production of IAA in planta. In the perennial rye grass variety, the addition 


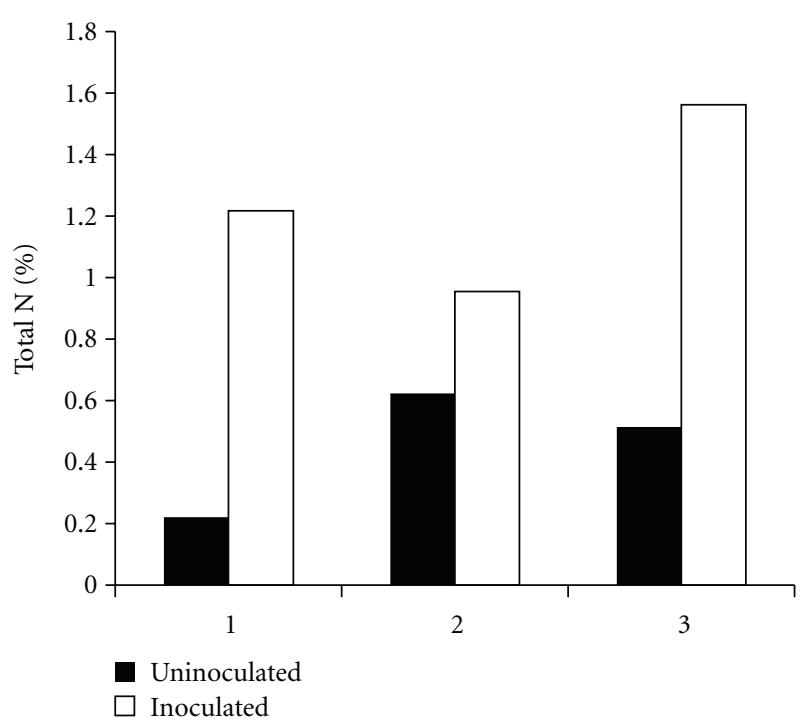

FIGURE 19: Perennial rye grass total nitrogen in roots. Total nitrogen content found in the roots of inoculated with endophytes:PTD1, WPB, WP19, WP1, and WW6 and uninoculated perennial grass variety $(\mathrm{G} 3)$ at 7 weeks in the various nitrogen treatments. 1: no nitrogen, 2: full nitrogen, and 3: 1/5 nitrogen.

of endophytes provided several benefits in the presence of nitrogen and water stresses. There was an earlier rooting response in the inoculated grasses, which would be beneficial to grasses that are planted in soils that have limited access to water and high bulk densities due to compaction by construction and agriculture. Interestingly, this grass variety responded exceptionally well to the endophyte consortia in fixing nitrogen after receiving a limited supply of fertilizer. The requirement of some nitrogen may have been due to the need for the endophyte to produce sufficient nitrogenase enzyme to begin nitrogen fixation.

However, not all plant crop species interacted positively with the endophyte treatment, indicating that the interaction is somewhat specific. Cultivar specificity was also noticed by other authors. In a study by Dong et al. [20], the authors showed that inoculation with a nitrogen fixing endophyte was cultivar-specific. In their experiments carried out with different varieties of wheat, only the Trenton variety responded positively to the inoculation with the endophyte where it relieved nitrogen deficiency symptoms and increased the total $\mathrm{N}$ concentration in the plant, whereas inoculation with other varieties resulted in no effect. In a study by Long et al. [34], the authors showed that inoculation of endophytes with plant growth promoting properties into nonhost plants elicited a species specific response. The authors reported different growth and fitness responses of different plant species to the same bacterial strains. In another study [35], inoculation effects of bacteria were studied on different varieties of corn with only a few varieties showing a positive response while others were nonresponsive. Other researchers have performed bacterial endophyte colonization studies with corn and other agronomic crops.
Zinniel et al. [36] conducted a host range study on endophytes obtained from monocots and showed that the endophytes colonized both monocots and dicot plant species.

The endophyte strain and plant cultivar specificities observed in our study as well as other studies point to the necessity of further research to understand the plant-microbe signaling necessary for effective colonizing and plant growth promoting capabilities. The level at which the interaction is blocked has yet to be determined. Experiments with fluorescently tagged endophytes could be performed to assess if the block is at entry into the plant or in colonization. Studies of the plant defense response are needed to determine if incompatible plant varieties are sustaining a defense response against the endophyte, thereby preventing its effective colonization. An understanding of these interactions may improve the rate of successful application of these beneficial endophytes.

\section{Conclusion}

Overall, this study demonstrated the successful colonization, growth enhancement, and increased fruit yields of certain crops by the addition of poplar and willow endophytes under greenhouse conditions. The absence of the inoculated endophyte in the fruits or seeds demonstrates that the endophyte does not appear to have the potential to enter the human diet. In rye grass, we also demonstrated evidence of nitrogen fixation due to inoculation with a mixture of endophytes. Future studies should be directed towards the potential use of these strains for crop development and fruit yield enhancements under poor nutrient conditions in field studies. However, since the response was specific, preliminary screening tests on each variety of crop with a variety of endophytes are essential before the system is ready for field application. Regardless, these studies demonstrate the potential benefits that endophytes may impart on crop plants which will benefit not only agriculture, but society at large.

\section{Acknowledgments}

The authors thank Professor Soo-Hyung Kim for his excellent advice and allowing us to use the greenhouse space at $\mathrm{CUH}$, and the students Alex Ching, Travis Lee, Patrick Walters, and Monica Fischer for their help with the measurements. This work was funded by the University of Washington Center for Commercialization.

\section{References}

[1] O. C. Bøckman, "Fertilizers and biological nitrogen fixation as sources of plant nutrients: perspectives for future agriculture," Plant and Soil, vol. 194, no. 1-2, pp. 11-14, 1997.

[2] A. V. Sturz and J. Nowak, "Endophytic communities of rhizobacteria and the strategies required to create yield enhancing associations with crops," Applied Soil Ecology, vol. 15, no. 2, pp. 183-190, 2000.

[3] J. Hallmann, A. Quadt-Hallmann, W. F. Mahaffee, and J. W. Kloepper, "Bacterial endophytes in agricultural crops," Canadian Journal of Microbiology, vol. 43, no. 10, pp. 895-914, 1997. 
[4] A. V. Sturz, B. R. Christie, B. G. Matheson, and J. Nowak, "Biodiversity of endophytic bacteria which colonize red clover nodules, roots, stems and foliage and their influence on host growth," Biology and Fertility of Soils, vol. 25, no. 1, pp. 13-19, 1997.

[5] M. A. Surette, A. V. Sturz, R. R. Lada, and J. Nowak, "Bacterial endophytes in processing carrots (Daucus carota L. var. sativus): their localization, population density, biodiversity and their effects on plant growth," Plant and Soil, vol. 253, no. 2, pp. 381-390, 2003.

[6] A. H. Sziderics, F. Rasche, F. Trognitz, A. Sessitsch, and E. Wilhelm, "Bacterial endophytes contribute to abiotic stress adaptation in pepper plants (Capsicum annuum L.)," Canadian Journal of Microbiology, vol. 53, no. 11, pp. 1195-1202, 2007.

[7] M. Beyeler, C. Keel, P. Michaux, and D. Haas, "Enhanced production of indole-3-acetic acid by a genetically modified strain of Pseudomonas fluorescens $\mathrm{CHA} 0$ affects root growth of cucumber, but does not improve protection of the plant against Pythium root rot," FEMS Microbiology Ecology, vol. 28, no. 3, pp. 225-233, 1999.

[8] S. Timmusk, B. Nicander, U. Granhall, and E. Tillberg, "Cytokinin production by Paenibacillus polymyxa," Soil Biology and Biochemistry, vol. 31, no. 13, pp. 1847-1852, 1999.

[9] R. S. Redman, Y. O. Kim, C. J. D. A. Woodward et al., "Increased fitness of rice plants to abiotic stress via habitat adapted symbiosis: a strategy for mitigating impacts of climate change," PLOS ONE, vol. 6, no. 7, Article ID e14823, 2011.

[10] B. Reiter, H. Bürgmann, K. Burg, and A. Sessitsch, "Endophytic nifH gene diversity in African sweet potato," Canadian Journal of Microbiology, vol. 49, no. 9, pp. 549-555, 2003.

[11] J. K. Vessey, "Plant growth promoting rhizobacteria as biofertilizers," Plant and Soil, vol. 255, no. 2, pp. 571-586, 2003.

[12] S. L. Doty, "Nitrogen-fixing endophytic bacteria for improved plant growth," in Bacteria in Agrobiology: Plant Growth Responses, D. K. Maheshwari, Ed., chapter 9, pp. 183-199, Springer, New York, NY, USA, 2011.

[13] M. Flores-Encarnación, M. Contreras-Zentella, L. Soto-Urzua, G. R. Aguilar, B. E. Baca, and J. E. Escamilla, "The respiratory system and diazotrophic activity of Acetobacter diazotrophicus PAL5," Journal of Bacteriology, vol. 181, no. 22, pp. 6987-6995, 1999.

[14] J. R. Gallon, "Reconciling the incompatible- $\mathrm{N}_{2}$ fixation and $\mathrm{O}_{2}$," New Phytologist, vol. 122, no. 4, pp. 571-609, 1992.

[15] M. Sevilla, R. H. Burris, N. Gunapala, and C. Kennedy, "Comparison of benefit to sugarcane plant growth and $15 \mathrm{~N} 2$ incorporation following inoculation of sterile plants with acetobacter diazotrophicus wild-type and Nif- mutant strains," Molecular Plant-Microbe Interactions, vol. 14, no. 3, pp. 358-366, 2001.

[16] A. Elbeltagy, K. Nishioka, T. Sato et al., "Endophytic colonization and in planta nitrogen fixation by a Herbaspirillum sp. Isolated from wild rice species," Applied and Environmental Microbiology, vol. 67, no. 3-12, pp. 5285-5293, 2001.

[17] V. L. D. Baldani and J. Döbereiner, "Host-plant specificity in the infection of cereals with Azospirillum spp," Soil Biology and Biochemistry, vol. 12, no. 4, pp. 433-439, 1980.

[18] S. Urquiaga, K. H. S. Cruz, and R. M. Boddey, "Contribution of nitrogen fixation to sugar cane. Nitrogen-15 and nitrogenbalance estimates," Soil Science Society of America Journal, vol. 56, no. 1, pp. 105-114, 1992.

[19] J. R. Stoltzfus, R. So, P. P. Malarvithi, J. K. Ladha, and F. J. De Bruijn, "Isolation of endophytic bacteria from rice and assessment of their potential for supplying rice with biologically fixed nitrogen," Plant and Soil, vol. 194, no. 1-2, pp. 25-36, 1997.

[20] Y. Dong, A. L. Iniguez, and E. W. Triplett, "Quantitative assessments of the host range and strain specificity of endophytic colonization by Klebsiella pneumoniae 342," Plant and Soil, vol. 257, no. 1, pp. 49-59, 2003.

[21] E. Martinez-Romero, T. Jimenez-Salgado, L. E. FuentesRamirez, A. Tapia-Hernandez, M. A. Mascarua-Esparza, and J. Caballero-Mellado, "Coffea Arabica L., a new host plant for Acetobacter diazotrophicus, and isolation of other nitrogenfixing acetobacteria," Applied and Environmental Microbiology, vol. 63, no. 9, pp. 3676-3683, 1997.

[22] S. Gagne, C. Richard, H. Rousseau, and H. Antoun, "Xylemresiding bacteria in alfalfa roots," Canadian Journal of Microbiology, vol. 33, no. 11, pp. 996-1000, 1987.

[23] D. Y. Kobayashi and D. J. Palumbo, "Bacterial endophytes and their effects on plants and uses in agriculture," in Microbial Endophytes, C. W. Bacon and J. F. White, Eds., pp. 199-233, Springer, New York, NY, USA, 2000.

[24] Z. Khan and S. L. Doty, "Characterization of bacterial endophytes of sweet potato plants," Plant and Soil, pp. 1-11, 2009.

[25] F. Rasche, R. Trondl, C. Naglreiter, T. G. Reichenauer, and A. Sessitsch, "Chilling and cultivar type affect the diversity of bacterial endophytes colonizing sweet pepper (Capsicum anuum L.)," Canadian Journal of Microbiology, vol. 52, no. 11, pp. 1036-1045, 2006.

[26] P. Nejad and P. A. Johnson, "Endophytic bacteria induce growth promotion and wilt disease suppression in oilseed rape and tomato," Biological Control, vol. 18, no. 3, pp. 208-215, 2000.

[27] G.V.M. De Pereira, K.T. Magalhaes, E.R. Lorenzetii, T.P. Souza, and R.F. Schwan, "A multiphasic approach for the identification of endophytic bacterial in strawberry fruit and their potential for plant growth promotion," Microbial Ecology, vol. 63, no. 2, pp. 405-417, 2012.

[28] S. L. Doty, "Growth-promoting endophytic fungi of forest trees," in Endophytes of Forest Trees: Biology and Applications, A. M. Pirttila and A. C. Carolin, Eds., Springer, New York, NY, USA, 2011.

[29] S. L. Doty, B. Oakley, G. Xin et al., "Diazotrophic endophytes of native black cottonwood and willow," Symbiosis, vol. 47, no. 1, pp. 23-33, 2009.

[30] G. Xin, G. Zhang, J. W. Kang, J. T. Staley, and S. L. Doty, "A diazotrophic, indole-3-acetic acid-producing endophyte from wild cottonwood," Biology and Fertility of Soils, vol. 45, no. 6, pp. 669-674, 2009.

[31] J. Knoth, S. H. Kim, G. Ettl, Doty, and S. L, "Effects of cross host species inoculation of nitrogen-fixing endophytes on growth and leaf physiology of maize," GCB Bioenergy. In press.

[32] R. J. Rennie, "A single medium for the isolation of acetylenereducing (dinitrogen-fixing) bacteria from soils," Canadian Journal of Microbiology, vol. 27, no. 1, pp. 8-14, 1981.

[33] M. D. Chilton, T. C. Currier, and S. K. Farrand, "Agrobacterium tumefaciens DNA and PS8 bacteriophage DNA not detected in crown gall tumors," Proceedings of the National Academy of Sciences of the United States of America, vol. 71, no. 9, pp. 3672-3676, 1974.

[34] H. H. Long, D. D. Schmidt, and I. T. Baldwin, "Native bacterial endophytes promote host growth in a species-specific manner; phytohormone manipulations do not result in common growth responses," PLoS ONE, vol. 3, no. 7, Article ID e2702, 2008 . 
[35] S. Mehnaz and G. Lazarovits, "Inoculation effects of Pseudomonas putida, Gluconacetobacter azotocaptans, and Azospirillum lipoferum on corn plant growth under greenhouse conditions," Microbial Ecology, vol. 51, no. 3, pp. 326-335, 2006.

[36] D. K. Zinniel, P. Lambrecht, N. B. Harris et al., "Isolation and characterization of endophytic colonizing bacteria from agronomic crops and prairie plants," Applied and Environmental Microbiology, vol. 68, no. 5, pp. 2198-2208, 2002. 


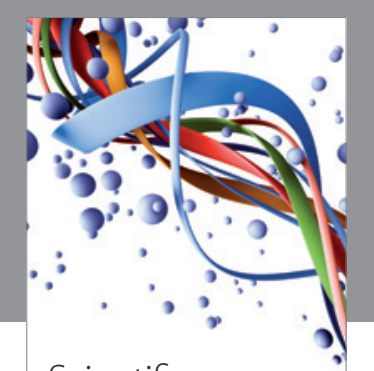

Scientifica
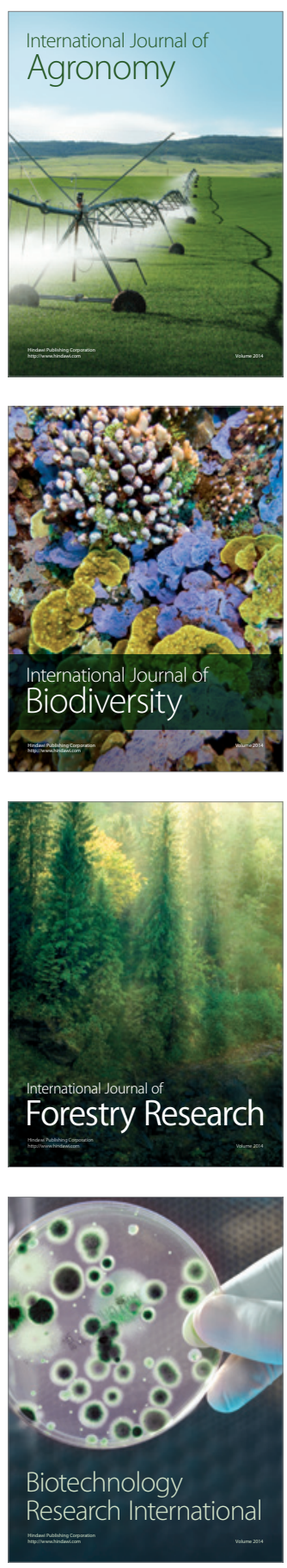
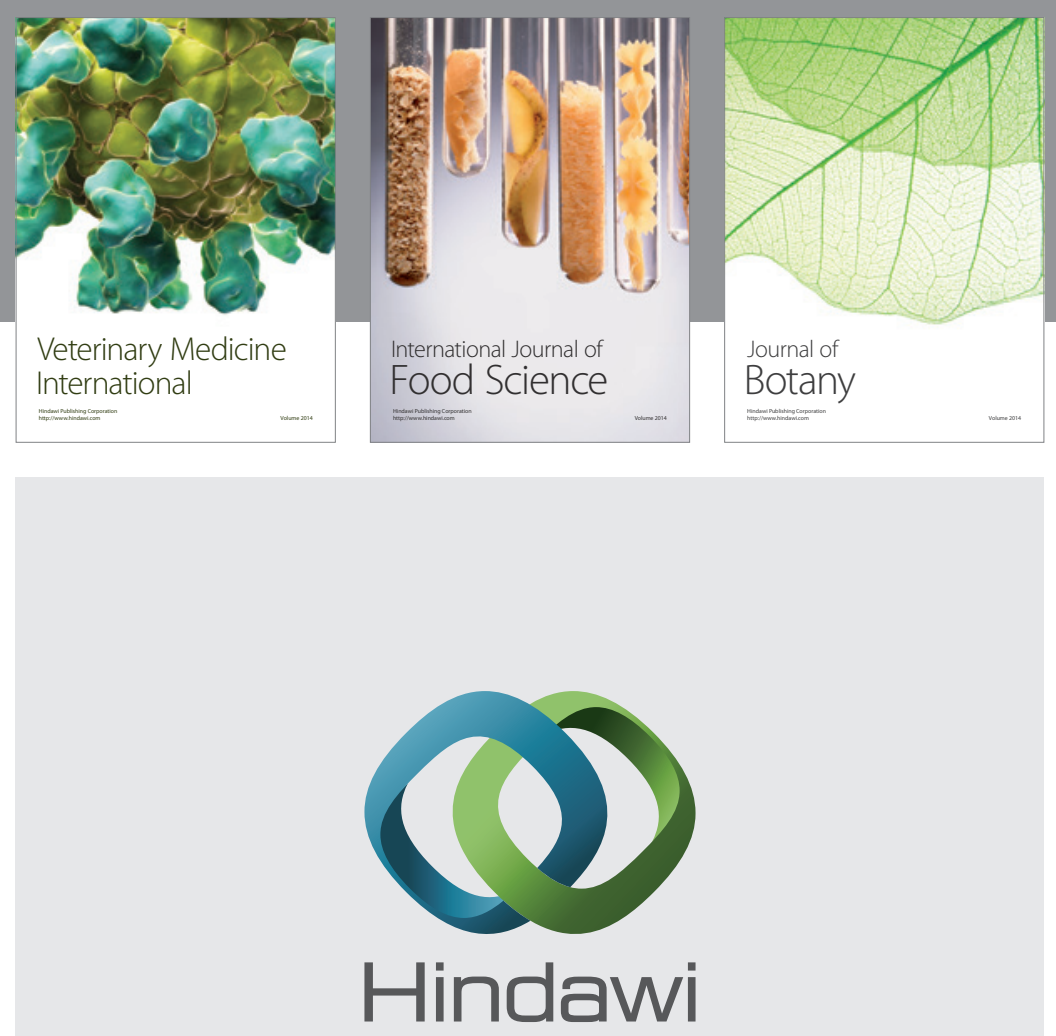

Submit your manuscripts at

http://www.hindawi.com
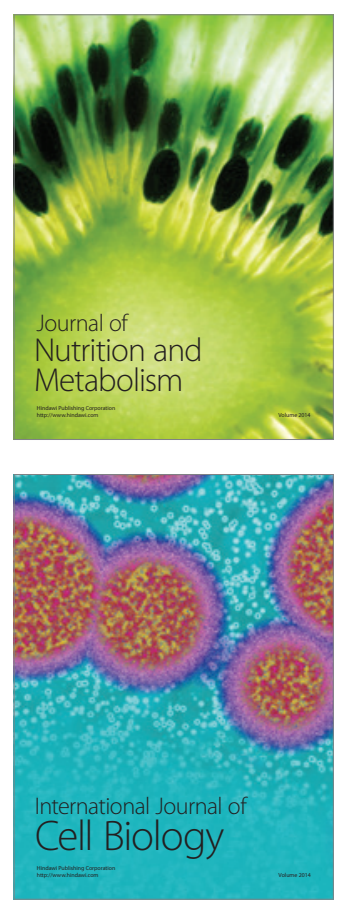
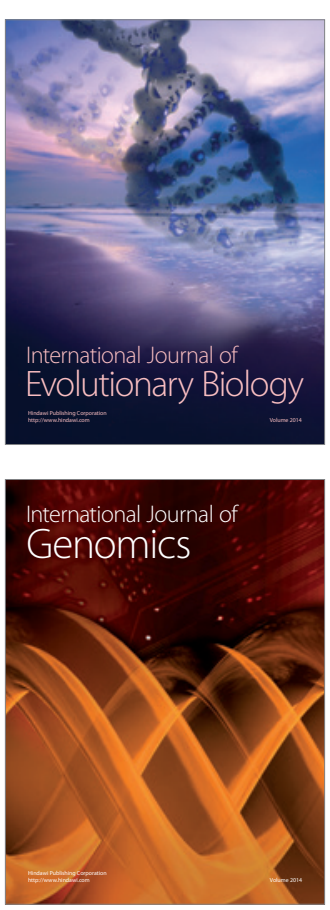
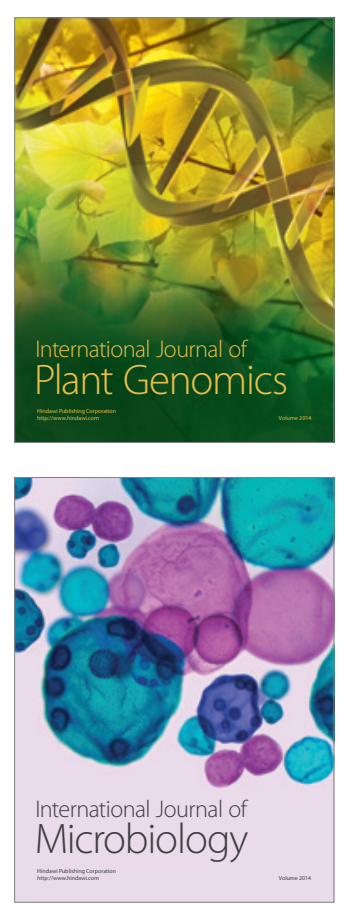

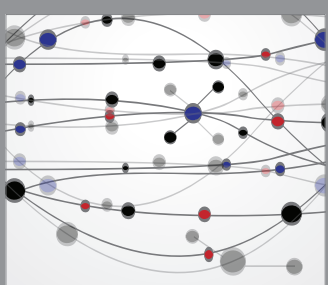

The Scientific World Journal
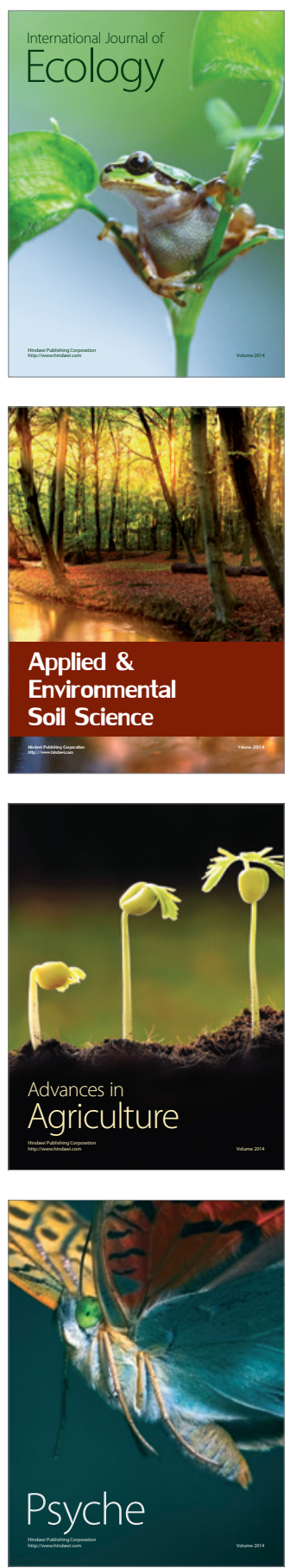